\title{
New Method for the Tracing of Power Transformers and Wires in Distribution Systems
}

\author{
Heejung Byun ${ }^{1,}$, , Sugoog Shon ${ }^{1, b^{*}}$ \\ ${ }^{1}$ Department of IT Engineering, The University of Suwon, IT Building, san 2-2 Wau-ri, \\ Bongdam-eup, Hwaseong-si,, Korea \\ aheejungbyun@suwon.ac.kr, bsshon@suwon.ac.kr (Corresponding)
}

Keywords: Power Transformer, Power Line Carrier, Micro Processor

\begin{abstract}
In modern cities, power distribution systems are very complicated systems consisted of 3-phase lines, 3-phase transformers, electric poles, etc. and those facilities are distributed among lots of buildings, roads, and trees. Tracing technology of transformers and wires is a challenging issue. Power line carrier communication is applied to trace transformers and wires in power distribution systems. Power transformer and wires have only a limited ability to carry higher frequencies. A new wire tracing system is proposed to utilize its limited propagation characteristics. The wire and transformer tracing system is designed and implemented based on a client-server with power line carrier communication function. Some simulation and experiments are conducted to understand and verify the theoretical concepts and practical characteristics.
\end{abstract}

\section{Introduction}

Electrical power systems are usually classified into high, medium and low voltage systems. High voltage systems transports energy at over $100 \mathrm{kV}$ from generating power plants to electrical substations located near demand centers. Medium voltage (typically below 50kV) and low voltage systems (less than $1 \mathrm{kV}$ ) deliver electricity from the substation to consumers. Various distribution topologies exist and differ from country to country, and also within each country, for example radial and interconnected distribution networks, delta and wye three-phase services, single-phase and split-phase low-voltage services, different earth systems. Usually in a substation, electric power is provided to end customers by means of $\mathrm{A}, \mathrm{B}, \mathrm{C}$ three phases through three or four wire conductors. From MV/LV transformer on electric pole or pad, electric power is serviced one or three phases through two, three or four wire conductors [1].

As electrician approaches toward the end of the distribution lines, the effort to distinguish both an absolute phase value and wire configurations to MV/LV transformer becomes more difficult. Electrician on site should correctly figure out electrical configurations such as phases and wire connections. Its wrong selection can result in fatal consequences for the operator and cause the loss of supply for the connected customers. Thus, electrician must become able to confirm there in the field as to which phase of A, B or C phases the wire conductors belong and as to which transformer the wire conductors are connected as soon as possible.

The unmistakable identification of wires and absolute phases is an absolute safety related task. When confusion for phases and wires occurs, disproportionate concentrations of loads can easily occur, and this type of disproportionate concentration of loads can cause phase unbalance that can lead to power loss or power failures and, further, equipment failures due to excessive voltage regulation or reduction of useful life, etc. What this would all mean is that it can result in substantial management difficulties such as economic losses, all because of the reduced quality of voltage supplied to the customer [2].

Consider the situation that many transformers and distribution lines are deployed along the street as shown in Fig. 1. There, an electric technician wants to find out transformer or distribution line configuration to which premise power line wires are connected. It is not easy to figure out because there are too many obstacles such as buildings, trees, or hills. 


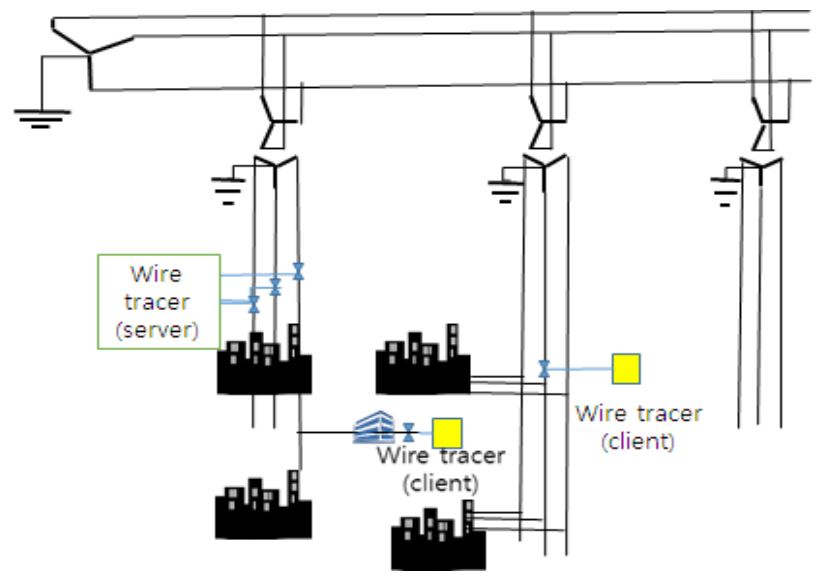

Fig. 1 Situation to figure out transformer and wire configurations.

The need to determine phases and wires is increasing. As such, most of phase distinguishing methods employ methods that distinguish phases based on comparing the known phase value of a substation with the unknown phase value of a local site [3-8]. The determination of an absolute phase is difficult when it is needed on site. For the most recent research of the phase determination, GPS technology is utilized to synchronize time at different locations, which is used to measure the phase for electric signals [2].

In addition to the phase determination, the wire tracing method is also a challenging issue. Also, electrician wants to make sure that some customers are serviced from a certain transformer. The determination of wire and transformer connectivity is difficult when it is needed on site. $\mathrm{Ku}$, etc. also has published the paper to identify customers served by transformer using PLC, but their method cannot support tracing and discrimination ability among the wires [9].

In this paper, we propose how to find out the wire and transformer tracing by using power-line communication for the easy, reliable and safe selection of wires. The paper uses some facts that power wire circuits have only a limited ability to carry higher frequencies. A new wire and transformer identification system is designed and implemented. The system consists of a transmitter and a receiver with power-line communication module. Some experiments are conducted to verify the theoretical concepts. Also some simulations are done to help and understand the proposed system by using Simulink simulator.

\section{Proposal of the Wire and Transformer Tracing System}

In the first, some backgrounds are investigated about the way to trace wires at local site. The recent wire tracing system, newly developed, consists of current impulse generator and receiver. The receiver is connected by a clamp for decoupling the identification signal. The pulse generator generates some special type of pulses and transmits them into the wires being traced [10]. The sent impulse signal causes an electromagnetic field with a defined polarity around the wire which is received with the flex coupler of the receiver, automatically synchronized. The directional clamp in combination with the parameter monitoring by the receiver provides a safe selection regardless of any interference. It means that only one conductor or wire has the correct polarity while all other cables have the opposite polarity. As another method, direct signals are injected on the transmitter and the received signals are analyzed in terms of amplitude-time-phase at receiver [11]. Another method is to communicate coded messages between central device and line device through the distribution networks [12].

Power Line Communication (PLC) is a technology that exchanges data via the AC electric power line. The PLC uses the existing electrical lines as communication medium, which provides the major benefit of eliminating considerable costs of installing networking infrastructure, like dedicated wires or antennas. Data is sent on the power lines by superposing a modulated high-frequency carrier signal on the line voltage, being high, medium or low, AC or DC. The carrier signal is then de-coupled and demodulated at the receiving end to recover the information. 


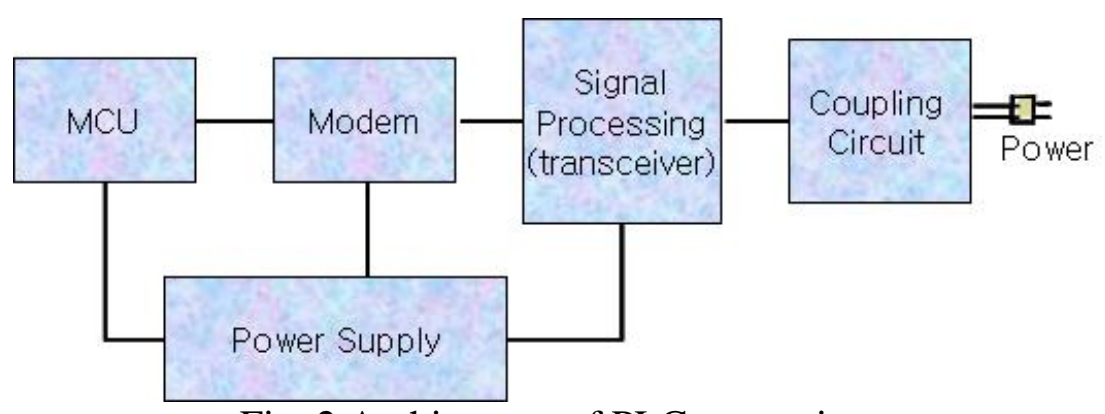

Fig. 2 Architecture of PLC transceiver.

Narrowband power line communication (N-PLC) is generally defined as communication over power line that is typically operating in transmission frequencies of up to $500 \mathrm{kHz}$ (as opposed to Broadband PLC that targets much higher bandwidth at shorter distances and operates over a much higher frequency band). For PLC modem, narrow band operating in the very low frequency/low frequency/medium frequency bands $(3-500 \mathrm{kHz})$, which include the European CENELEC bands (3-148.5 kHz), the US FCC band (10-490 kHz), the Japanese ARIB band (10-450 kHz), and the Chinese band (3-500 kHz) can be adopted.

Whatever the type of modulation used, a PLC transceiver includes a few basic blocks as shown in Fig. 2. Different solutions use various levels of integration of these components, from full-digital modems with external discrete components to highly integrated systems-on-chip [11-13]. To transmit or receive data from the electric power line, connect a live line with PLC modem through the coupling circuit.

Ku's and Shon's methods cannot discriminate wires of 3-phase distribution lines, instead, it is possible to discriminate transformer served to customers [2, 9]. Fig. 3 shows the architecture of a wire and transformer tracing system, which is comprised of a server and a client. Message from client program is transferred into PLC modem through interface. PLC modem converts the message into signal, which is modulated into higher carrier frequency. The modulated signal is transferred into the server. The server side's PLC modem receives the modulated signal and demodulates that into data. Then it is moved into the microprocessor. The server program responds to the client. Especially, the server has multiple PLC modems instead of one modem. Each modem will communicate with the client and send different coded message, which is used to identify each phase.

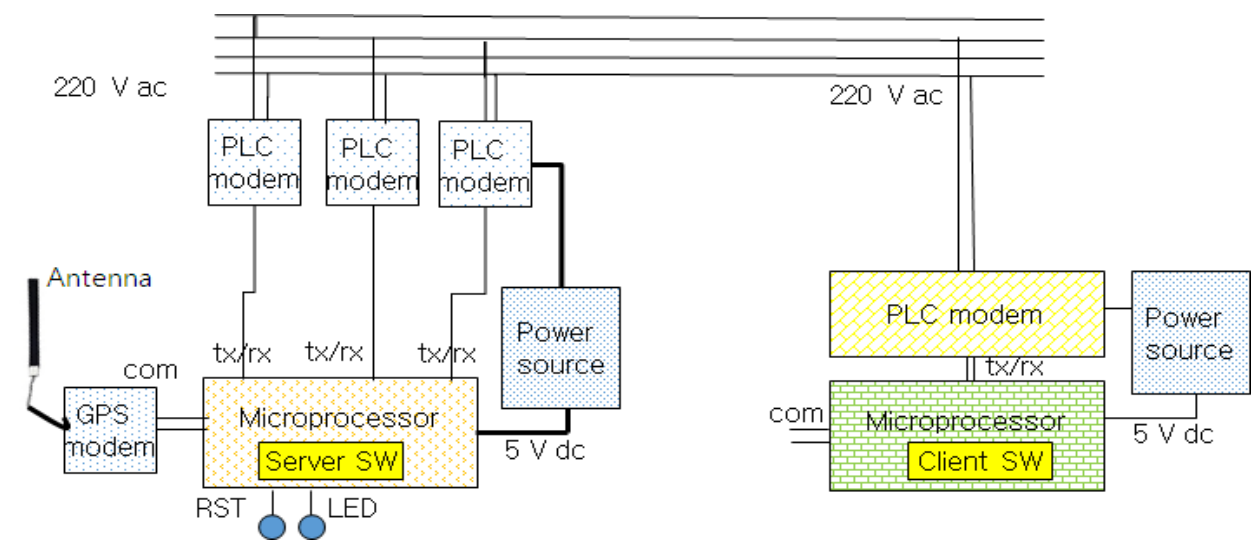

Fig. 3 Architecture of proposed wire and transformer tracing system.

\section{Simulation and Experimental Results}

For its application to the wire and transformer tracing technology, characteristics of higher frequency carrier propagation are analyzed through several transformers with Simulink. Simulink models are presented for the analyses. Using the sample distribution model, the distribution system is analyzed with Simulink when carrier signal of $290 \mathrm{kHz}$ and $300 \mathrm{~mA}$ is injected onto the distribution lines. 
As an example, the Simulink model including several transformers and distribution lines is shown in Fig. 4. More details for the Simulink model such as power sources, lines, unbalanced loads, transformers, grounding resister and phase measurement system for phase simulation are simply explained below [2]. In the first, the input source is expressed in terms of power with an internal resistance and inductance. Power capacity that can sufficiently provide power used in the load is arranged. Power is wired as a 3-phase and a supply voltage is sinusoidal. A neutral point is connected to a grounding resistance. The power output is provided to distribution lines through a transformer wired as a wye $(Y)$ or delta $(\Delta)$.

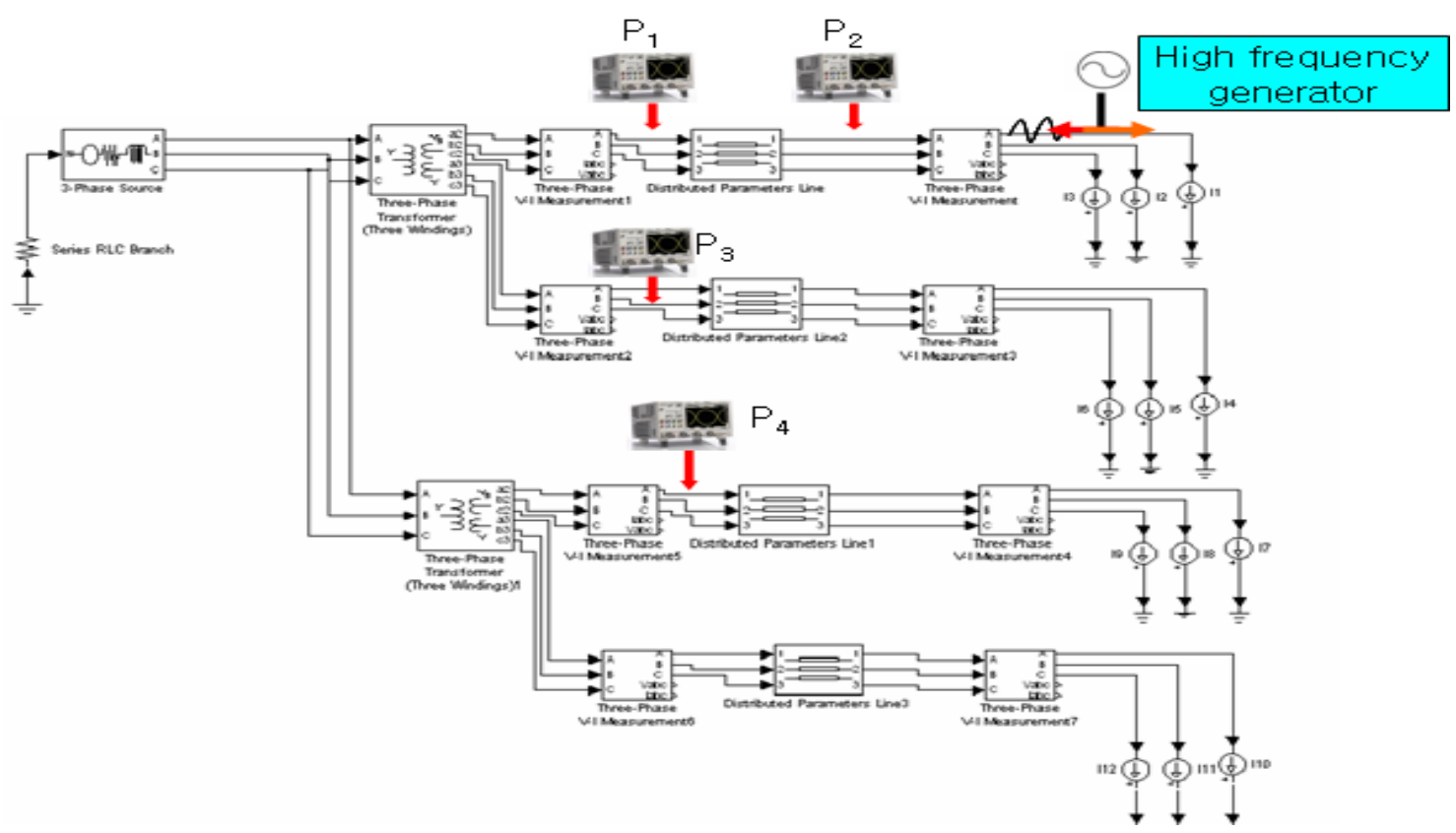

Fig. 4 Sample of Simulink distribution system model.

In order to show the characteristics of modulated signal, some larger carrier signal of $300 \mathrm{~mA}$ is adopted even though it is bigger than usual. In general, the primary side of the transformer is high voltage of $22.9 \mathrm{kV}$ and the secondary side is $220 \mathrm{~V}$. So the turn ratio between the primary and the secondary is about $100(=22.9 \mathrm{kV} / 220 \mathrm{~V})$. The amplitude of the modulated signal injected from the secondary becomes much lesser. We can see that modulated signal is gone away at the primary side. Fig. 5 shows the modulated voltage waveform for three different phases injected at the secondary side. Theoretical simulations result in that the injected carrier signal is hard to cross over the higher level transformer as shown in Fig. 6.

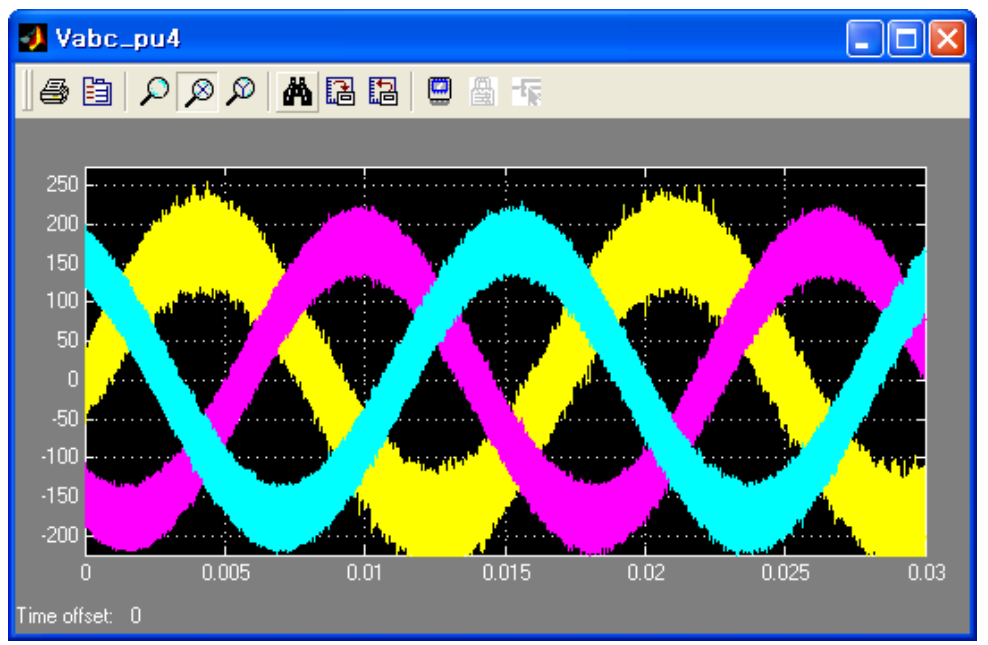

Fig. 5 Modulated voltage waveform with the carrier signal of $290 \mathrm{kHz}$ and $300 \mathrm{~mA}$. 


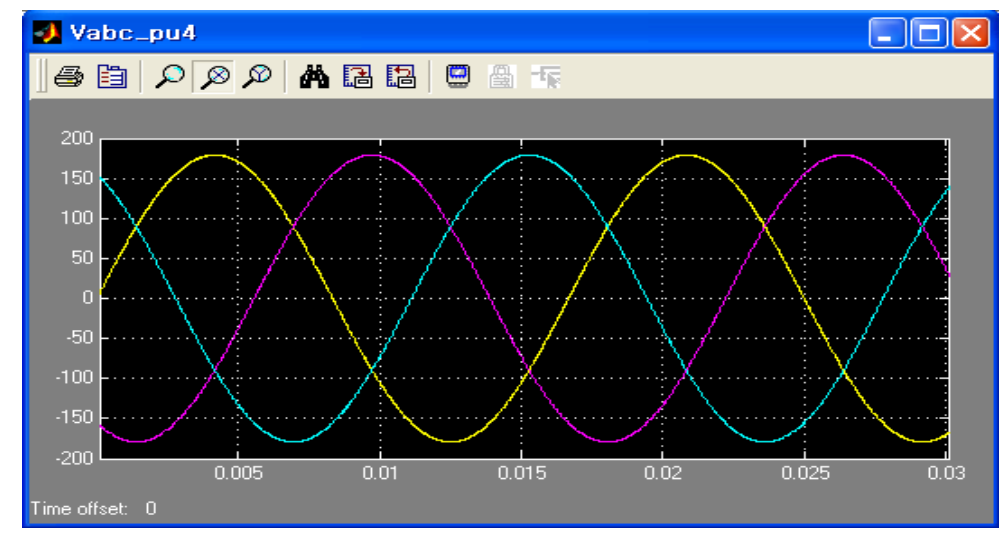

Fig. 6 The secondary-side low voltage waveform at P4 of the other transformer cross one transformer for carrier signal of $290 \mathrm{kHz}$ and $300 \mathrm{~mA}$.

A test system is implemented with the PLC modem chip having carrier frequency of $290 \mathrm{kHz} /$ $125 \mathrm{kHz}$. Specifically, frequencies of $148.5 \mathrm{kHz}$ and less have been recognized by Europe's CENELEC standards body for use in N-PLC systems on a public utility's power wires. Within this frequency range the resulting data rates are modest, ranging from $1 \mathrm{Kbps}$ to less than $100 \mathrm{Kbps}$. These rates are appropriate for telemetry and control applications. Arduino Uno board is used for microprocessor unit. Experiments show that the PLC data after modulation at the client is transferred into the range of PLC carrier propagation as shown in Fig. 7.

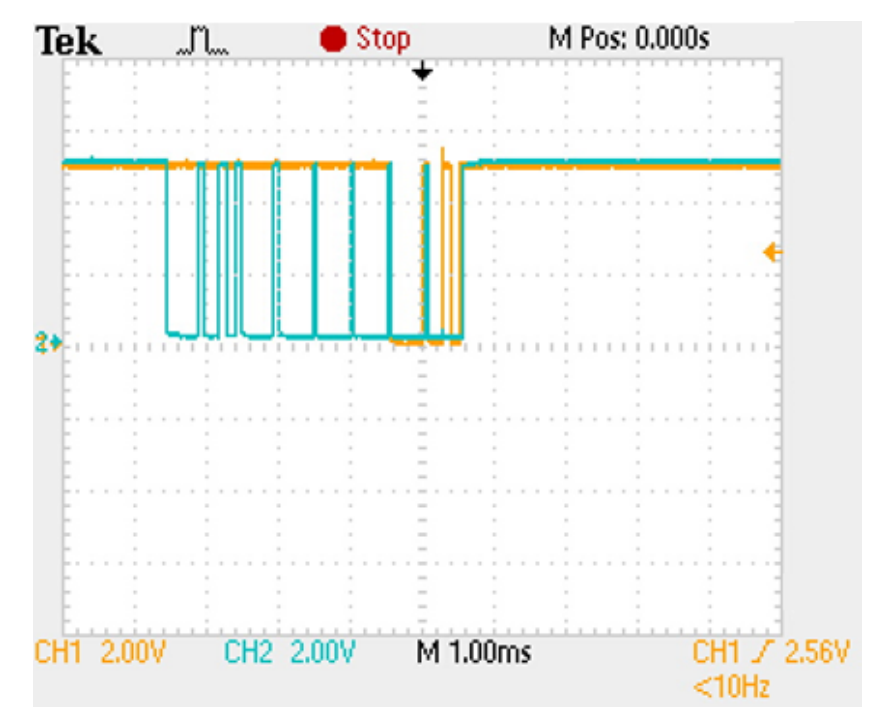

Fig. 7. PLC data communication after demodulation.

\section{Conclusions}

In order to verify the idea, the proposed wire and transformer tracing system is implemented with narrowband power-line communication module with simple MAC and $290 \mathrm{kHz}$ and $125 \mathrm{kHz}$. Experiments show also that the carrier signal is not transferred over the higher level transformer.

In the last, we can conclude that the prototype method based on power line carrier technology can be utilized to figure out wire and transformer configurations in the complex distribution lines. For the further research topics, more experiments and algorithm to figure out wire tracing between client and server are considered.

\section{Acknowledgement}

This study was conducted by [GRRC (Gyeonggi Regional Research Center) Suwon 2015-B4, Development of smart emergency response and application systems] as part of projects of GRRC, Gyeonggi Province. The advice received from S. J. Choi of Editech, Ltd. is highly appreciated. 


\section{References}

[1] C. D. Scott, R. E. Smalley, Effects of Carbonyl Bond and Metal Cluster Dissociation and Evaporation Rates on Predictions of Nanotube Production in HiPco, J. Nanosci. Nanotechnol. 3 (2003) 75-79.

[2] H. J. Byun, S. G. Shon, Phase Shift Analysis and Phase Identification for Distribution System with 3-Phase Unbalanced Constant Current Loads, J. Electr. Eng. Technol. 8(4) (2013) 742-749.

[3] J. McDonald, Electric Power Substations Engineering, 2nd, CRC Press, 2007.

[4] B. Michel, Telephaseing method and system for remotely identifying unknown phases of transmission or distribution lines within an electrical network, US Patent 4626622, 1986.

[5] P. A. Lawrence, Apparatus and method for identifying the phase of a three phase power line at a remote location, US Patent 5510700, 1996.

[6] K.E. Martin, et al. IEEE Standard for Synchrophasers for Power Systems, IEEE Trans. Power Deliv. 13(1) (1998) 73-77.

[7] G. H. Piesinger, Apparatus and method for identifying cable phase in a three-phase power distribution network, US Patent 7,031,859, 2006.

[8] W. H. Kersting, Distribution System Modeling and Analysis, CRC Press, 2002.

[9] T. T. Ku, C. S. Chen, C. H. Lin, M. S. Kang, Identification of customers Served by Distribution Transformer using Power Line Carrier Technology, The $4^{\text {th }}$ IEEE Conference on Industrial Electronics and Applications, 25-27 May, ICIEA 2009, 2009, 3476-3481.

[10]http://www.sebakmt.com/products/cable-identifier.html

[11]www.powerpoint-engineering.com BAUR KSG 100 cable identifier

[12]F. L. Bertotti, M. S. Hara, P. J. Abatti, A simple method to measure phase difference between sinusoidal signals, Rev. Sci. Instrum. 81 (2010) 115106.

[13]C. Andrieu, E. Dauphant, D. Boss, A Frequency-Dependent Model For a MV/LV Transformer, IPST ’99- International Conference on Power Systems Transients, June 20-24, 1999. 